\title{
Optimized Controller Design for an Islanded Microgrid using Non-dominated Sorting Sine Cosine Algorithm (NSSCA)
}

\author{
Quazi Nafees Ul Islam \\ Electrical and Electronic \\ Engineering Department \\ Islamic University of Technology \\ Gazipur, Bangladesh \\ quazinafees@iut-dhaka.edu
}

\author{
Saad Mohammad Abdullah \\ Electrical and Electronic \\ Engineering Department \\ Islamic University of Technology \\ Gazipur, Bangladesh \\ saadabdullah@iut-dhaka.edu
}

\author{
Md. Arif Hossain \\ Electrical and Electronic \\ Engineering Department \\ Islamic University of Technology \\ Gazipur, Bangladesh \\ arifhossain@iut-dhaka.edu
}

\begin{abstract}
In order to cope with the increasing energy demand, microgrids emerged as a potential solution which allows the designer a lot of flexibility. The optimization of the controller parameters of a microgrid ensures a stable and environment friendly operation. Non-dominated Sorting Sine Cosine Algorithm (NSSCA) is a hybrid of Sine Cosine Algorithm and Non-dominated Sorting technique. This algorithm is applied to optimize the control parameters of a microgrid which incorporates both static and dynamic load. The obtained results are compared with the results of the established Non-dominated Sorting Genetic Algorithm-II (NSGA-II) in order to justify the proposal of the NSSCA. The average time needed to converge in NSSCA is $7.617 \mathrm{~s}$ whereas NSGA-II requires an average of $10.660 \mathrm{~s}$. Moreover, the required number of iterations for NSSCA is $\mathbf{2}$ which is significantly less in comparison to the $\mathbf{1 2}$ iterations in NSGA-II.
\end{abstract}

Keywords-multi-objective; NSGA-II; NSSCA; dynamic load; static load; SPSS;

\section{INTRODUCTION}

Renewable energy sources are often integrated in microgrids because they are environmentally friendly and are considered an answer to the fossil fuel scarcity. However, due to their unpredictable nature, renewable energy sources often hamper stability and may cause large frequency and voltage deviations in a microgrid [1]. The control parameters play a significant role for smooth and efficient operation of a microgrid. If there is any type of disturbance in the system, the selection of proper controller parameters and their tuning at optimized value ensures stable system operation [2]. Thus, research is now focused in optimizing the controller parameters, load sharing, cost etc. of a microgrid with a view to enhance its stability, efficiency, and cost effectiveness [3, 4]. In this aspect, various optimization algorithms are often adopted because they can often identify the global optimum system and also have a better convergence probability $[5,6]$. In Single Objective Optimization (SOO), the aim generally is to search for the best design or decision, which is expected to be the global solution of the optimization problem. But in the case of Multiple Objective Optimization (MOO), there may be one or more solutions which may be the best (global minimum or maximum) with respect to all objectives [7]. However, MOO renders a greater flexibility to the designers than SOO while selecting the most optimum result [8] because instead of presenting a single solution, MOO provides a set of solutions known as the Pareto front where none of the solutions dominates the others and thus the designer can choose any solution depending on his choice/requirements. Various works have been conducted in optimizing the controller parameters of a microgrid. In [9], artificial fish swarm algorithm was used to optimize only the droop controller gains for controlling the frequency deviation in a microgrid operating in islanded mode but it did not optimize other controller parameters. Authors in [10] used the MOO NSGA-II in optimizing the controller parameters but lacked comparison analysis between existing works. In this regard, the present study proposes a new MOO where Sine Cosine Algorithm (SCA) is combined with Nondominated Sorting technique to form the hybridized Nondominated Sorting Sine Cosine Algorithm (NSSCA). Basically SCA is an SOO which was first introduced in 2015 [11]. The incorporation of Non-dominated Sorting Technique transforms this SOO into an MOO. The proposed NSSCA is being used to obtain global optimum control parameters for an islanded microgrid consisting of both static and dynamic load. The main focus of this study is to obtain a better dynamic performance during load variation by applying NSSCA. In order to establish the efficacy of the designed NSSCA, the results are compared with the ones of the established Non-dominated Sorting Genetic Algorithm (NSGA-II) [12].

\section{MICROGRID MODEL}

In this study, an islanded microgrid as shown in Figure 1, composed of two Distributed Generation (DG) units where one unit has static (R-L) load installed and an induction motor on the other unit is considered as the dynamic load. The complete microgrid model used in this study is adopted from [2, 13]. In microgrid modeling, inverter, loads, and network design are the three main parts. Figure 2 shows the block diagram of an inverter connected to the microgrid along with its associated controllers. Among the three controller units: power controller determines the frequency and magnitude of the output voltage reference for the voltage controller, voltage controller 
determines the inductor output currents' reference using proportional integrator $(\mathrm{PI})$ regulator after comparing the actual and reference voltage values, and finally the current controller supplies switching signals to the inverter. There are thirteen states for individual inverter units, i.e. twenty-six states in total for the two inverters, two states for static load model, two states for line network, and five states for the induction motor. The complete microgrid model used in this study is developed by incorporating the state space model of individual inverter, static load model, line network, and induction motor. The total number of state variables is eight as shown in (1).

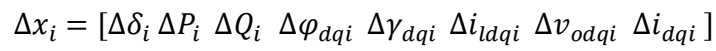

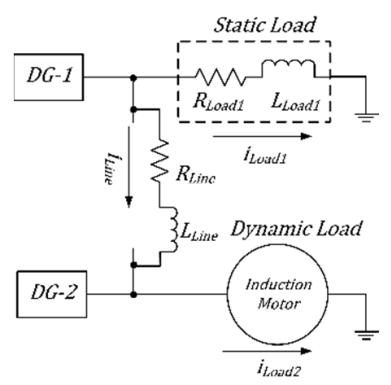

Fig. 1. Two DGs with static and dynamic load.

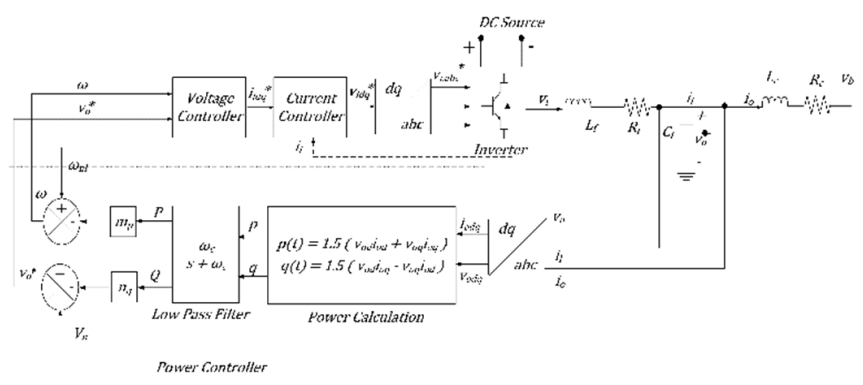

Fig. 2. Block diagram of an inverter connected to microgrid.

\section{PROBLEM FORMULATION}

Stable operation of a microgrid in islanded mode is an important aspect that needs to be ensured in order to acquire proper system output. The presence of both static and dynamic load in the microgrid creates challenges to its stable operation. Moreover, controller parameters play a vital role in system stability. In this system, PI regulators are used to vary gains of both voltage and current controllers. These controller gains need to be fine-tuned within proper limits for stable operation of the microgrid.

\section{A. Objective Function}

The microgrid model used in this study has eight controller gain parameters as there are two inverters for two DGs and each inverter has a separate voltage and current controller unit. Each voltage and current controller has separate PI regulators to control the controller gains. Here, $K_{p v 1}, K_{i v 1}$ and $K_{p v 2}, K_{i v 2}$ represent the PI gains of the voltage controller of inverter-1 and inverter-2 respectively. Similarly, $K_{p c 1}, K_{i c 1}$ and $K_{p c 2}, K_{i c 2}$ represent the PI gains of the current controller of inverter-1 and inverter-2 respectively. Eigenvalue analysis provides information regarding damping characteristics of a system which plays an important role in system stability. If the eigenvalues of the states of the microgrid model move away from the imaginary axis and go in the left half of the s-plane, their real part becomes more negative, the damping performance of the system is improved, and system stability is ensured. The main objective of this study is to optimize the above-mentioned controller gains to obtain a stable performance. The objective functions are mentioned in (2) and (3) where $\sigma$ and $\zeta$ indicate the real part of the eigenvalues and the damping ratio respectively.

$$
\begin{gathered}
\text { Minimize, } J_{1}=\left\{-\sigma_{\text {desired }}-\operatorname{minimum}\left(\sigma_{N}\right)\right\} \\
\text { Minimize, } J_{2}=\left\{\zeta_{\text {desired }}-\operatorname{minimum}\left(\zeta_{N}\right)\right\}
\end{gathered}
$$

$N$ represents the total number of states, which is 35 for this study. For each of these states the $\sigma$ and $\zeta$ values will be evaluated so that both the objectives are satisfied. $\sigma_{\text {desired }}$ and $\zeta_{\text {desired }}$ specify the limit of objective functions [14]. These two objective functions are contradictory in nature which can be understood from (4) where $\omega$ represents the frequency of the states. From (4) it can be seen that if the magnitude of $\sigma$ increases then $\zeta$ becomes more negative i.e. it reduces and vice versa.

$$
\zeta_{N}=\frac{-\sigma_{N}}{\sqrt{\sigma_{N}^{2}+\omega_{N}^{2}}}
$$

The constraint for this study is given in (5) where controller gains are limited to a desired boundary which is obtained by performing root locus analysis in order to obtain a stable microgrid system with improved damping performance.

$$
0<K_{p v 1,2}, K_{p i 1,2}, K_{i v 1,2}, K_{i c 1,2}<500
$$

\section{B. Proposed Solution}

NSSCA, a novel hybridized optimization algorithm, is proposed in this study. The optimization technique is presented with the help of the flow chart in Figure 3. The detailed steps of the algorithm are given below.

Step 1: Initialize the system parameters and generate the initial set of population. The total number of iterations is also defined in this step.

Step 2: The fitness of the solutions is evaluated by the objective functions $J_{1}$ and $J_{2}$.

Step 3: Non-dominated sorting of the initial generation of population is carried out on the basis of fitness value.

Step 4: In this step, crowding distance and ranking of the population is done.

Step 5: The position of all the solution sets is updated by (6) and (7) following [11].

$$
\begin{array}{ll}
X_{i}^{t+1}=X_{i}^{t}+r_{1} \times \sin \left(r_{2}\right) \times\left|r_{3} P_{i}^{t}-X_{i}^{t}\right|, & r_{4}<0.5 \\
X_{i}^{t+1}=X_{i}^{t}+r_{1} \times \cos \left(r_{2}\right) \times\left|r_{3} P_{i}^{t}-X_{i}^{t}\right|, & r_{4} \geq 0.5
\end{array}
$$

where $X_{i}^{t}$ is the current solution after the $t^{t h}$ iteration where the solution is along the $i^{\text {th }}$ dimension. Similarly $P_{i}^{t}$ is the destination solution point after the $t^{\text {th }}$ iteration along the $i^{\text {th }}$ 
dimension. Here, $r_{1}$ indicates the direction along which the solution will move, i.e. whether the solution will confine its movement within the space between the solution and the destination point or traverse beyond it. $r_{1}$ is updated using (8) where $a$ is a constant and $T$ is the maximum number of iterations.

$$
r_{1}=a-t \frac{a}{T}
$$

$r_{2}$ is a random number $[0,2 \pi]$ which indicates the distance of the movement of the solution inside or outside the destination and $r_{3}$ indicates a random weight in defining the effect of destination in distance calculation. If $r_{3}>1$ then the effect of the destination is emphasized and the opposite if $r_{3}<1$ [11]. $r_{4}$ is a random number between $[0,1]$ which indicates which of (6) or (7) should be followed to update the position.

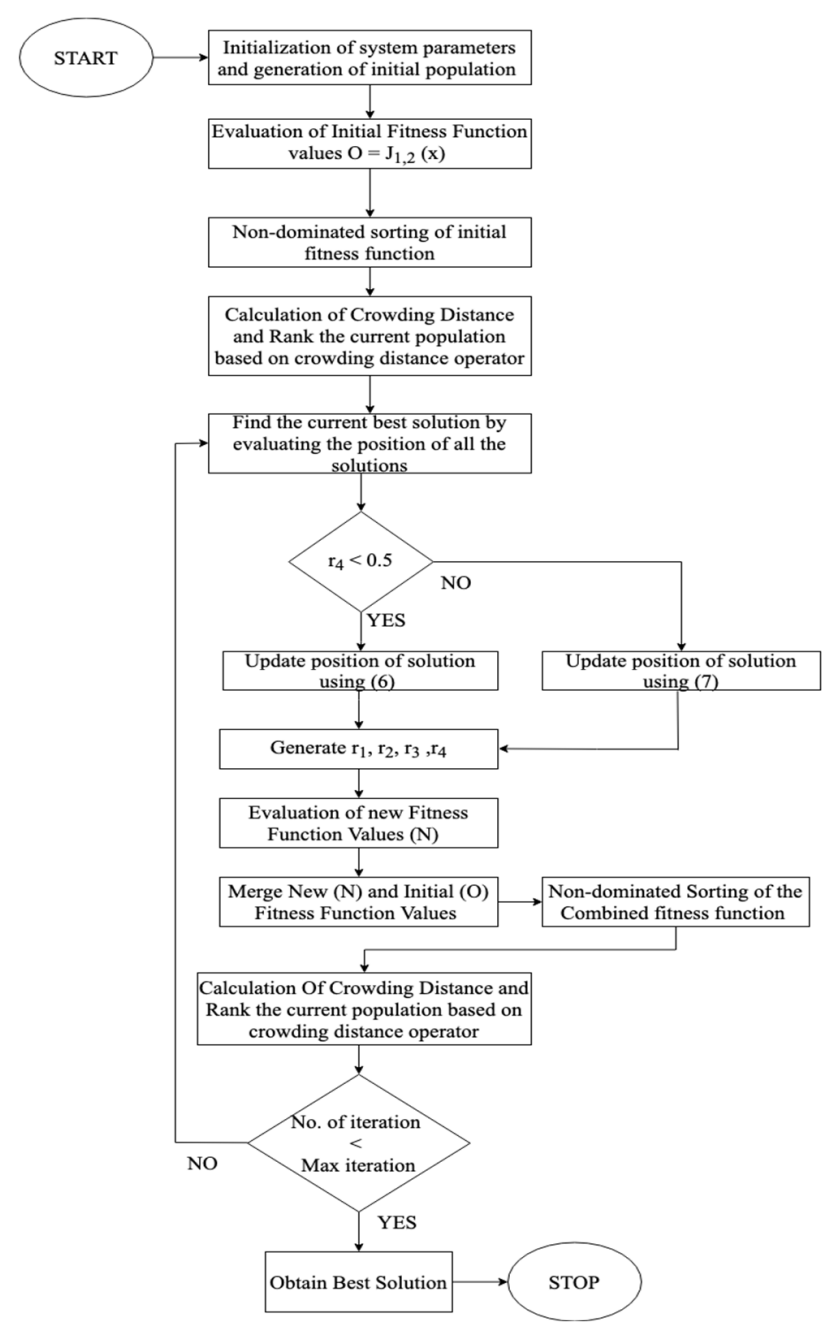

Fig. 3. Flowchart of the NSSCA.

Step 6: In order to update the position of the solutions $r_{1}, r_{2}, r_{3}$ and $r_{4}$ need to be updated to reach the best destination point and determine the best solution.

Step 7: Merge the new set of solution with the initial set of and then non-dominated sorting, crowding distance calculation and ranking of the merged set of solutions is applied until the maximum number of iterations is reached.

Step 8: The best possible position of the solution is determined after the final iteration and that position indicates the best solution

\section{RESULTS AND DISCUSSION}

\section{A. Eigenvalue Analysis}

The ability of the proposed NSSCA algorithm in stabilizing the system was examined through eigenvalue analysis. The eigenvalues of different states obtained before and after optimizing the controller parameters using NSSCA are shown in Table I. From the Table, it can be observed that for some of the states $\Delta \varphi_{q 2}, \Delta \gamma_{d 2}, \Delta i_{o q 2}$ and $\Delta i_{\text {LineD }}$ the eigenvalues are positive which indicates their location on the right side of the $s$ plane and thus instability to the system is introduced. After optimizing the controller parameters using NSSCA, it is observed that the obtained negative eigenvalues of the aforementioned states shifted their location from the right side to the left side of the $s$ plane making the system more stable.

TABLE I. EIGENVALUE ANALYSIS

\begin{tabular}{|c|c|c|c|}
\hline \multirow{2}{*}{ Index } & \multirow{2}{*}{ State } & \multicolumn{2}{|c|}{\begin{tabular}{|c|} 
Eigen value of the state \\
\end{tabular}} \\
\hline & & Before optimization & After optimization \\
\hline 1 & $\Delta \delta_{1}$ & $-2909410+12209388 i$ & $-2909410+12209388 \mathrm{i}$ \\
\hline 2 & $\Delta P_{1}$ & $-2909410-12209388 \mathrm{i}$ & $-2909410-12209388 \mathrm{i}$ \\
\hline 3 & $\Delta Q_{1}$ & $-3261123+8309127 i$ & $-3261123+8309127 i$ \\
\hline 4 & $\Delta \varphi_{d 1}$ & $-3261123-8309127 i$ & $-3261123-8309127 i$ \\
\hline 5 & $\Delta \varphi_{q 1}$ & $-55.194+45315 i$ & $-19853+389577 \mathrm{i}$ \\
\hline 6 & $\Delta \gamma_{d 1}$ & $-55.194-45315 \mathrm{i}$ & $-19853-389577 i$ \\
\hline 7 & $\Delta \gamma_{q 1}$ & $-352.417+44476.812 \mathrm{i}$ & $-19823+389227 \mathrm{i}$ \\
\hline 8 & $\Delta i_{l d 1}$ & $-352.417-44476.812 \mathrm{i}$ & $19823-389227 i$ \\
\hline 9 & $\Delta i_{l q 1}$ & $-4107.969+31524.405 i$ & $-29070+318066 \mathrm{i}$ \\
\hline 10 & $\Delta v_{\text {od } 1}$ & $-4107.969-31524.405 i$ & $-29070-318066 \mathrm{i}$ \\
\hline 11 & $\Delta v_{o q 1}$ & $-5629.972+29764.098 \mathrm{i}$ & $-29078+318482 \mathrm{i}$ \\
\hline 12 & $\Delta i_{\text {od } 1}$ & $-5629.972-29764.098 \mathrm{i}$ & $-29078-318482 \mathrm{i}$ \\
\hline 13 & $\Delta i_{o q 1}$ & $-8720.541+8365.113 i$ & $-8277.936+20647.906 \mathrm{i}$ \\
\hline 14 & $\Delta \delta_{2}$ & $-8720.541-8365.113 i$ & $-8277.936-20647.906 \mathrm{i}$ \\
\hline 15 & $\Delta P_{2}$ & $-6328.127+8624.675 i$ & $-12555.159+18762.199 \mathrm{i}$ \\
\hline 16 & $\Delta Q_{2}$ & $-6328.127-8624.675 i$ & $-12555.159-18762.199 \mathrm{i}$ \\
\hline 17 & $\Delta \varphi_{d 2}$ & $-1291.429+0 \mathrm{i}$ & $-2209.282+504.685 i$ \\
\hline 18 & $\Delta \varphi_{q 2}$ & $213.426+784.754 \mathrm{i}$ & $-2209.282-504.685 i$ \\
\hline 19 & $\Delta \gamma_{d 2}$ & $213.426-784.754 \mathrm{i}$ & $-25.460+198.321 \mathrm{i}$ \\
\hline 20 & $\Delta \gamma_{q 2}$ & $-81.284+376.280 \mathrm{i}$ & $-25.460-198.321 \mathrm{i}$ \\
\hline 21 & $\Delta i_{l d 2}$ & $-81.284-376.280 \mathrm{i}$ & $-157.538+0 \mathrm{i}$ \\
\hline 22 & $\Delta i_{l q 2}$ & $-162.677+0 \mathrm{i}$ & $-1.092+55.904 \mathrm{i}$ \\
\hline 23 & $\Delta v_{\text {od } 2}$ & $-70.227+1.578 \mathrm{i}$ & $-1.092-55.904 \mathrm{i}$ \\
\hline 24 & $\Delta v_{o q 2}$ & $-70.227-1.578 \mathrm{i}$ & $-70.694+0 \mathrm{i}$ \\
\hline 25 & $\Delta i_{o d 2}$ & $-67.497+0 \mathrm{i}$ & $-67.571+1.586 \mathrm{i}$ \\
\hline 26 & $\Delta i_{o q 2}$ & $22.647+0 \mathrm{i}$ & $-67.571-1.586 \mathrm{i}$ \\
\hline 27 & $\Delta i_{\text {LineD }}$ & $1.315+0 \mathrm{i}$ & $-2.187+0 \mathrm{i}$ \\
\hline 28 & $\Delta i_{\text {LineQ }}$ & $-2.394+0 \mathrm{i}$ & $-0.369+0.044 \mathrm{i}$ \\
\hline 29 & $\Delta i_{\text {LoadD }}$ & $-2.393+0 \mathrm{i}$ & $-0.369-0.044 i$ \\
\hline 30 & $\Delta i_{\text {LoadQ }}$ & $-0.018+0.045 i$ & $-1.686+0.009 i$ \\
\hline 31 & $\Delta i_{Q s}$ & $-0.018-0.045 \mathrm{i}$ & $-1.686-0.009 \mathrm{i}$ \\
\hline 32 & $\Delta i_{D S}$ & $-0.021+0 \mathrm{i}$ & $-1.686+0 \mathrm{i}$ \\
\hline 33 & $\Delta i_{Q r}$ & $-0.329+0 \mathrm{i}$ & $-0.560+0 \mathrm{i}$ \\
\hline 34 & $\Delta i_{D r}$ & $-0.200+0 \mathrm{i}$ & $-0.701+0 \mathrm{i}$ \\
\hline 35 & $\Delta s_{L}$ & $-0.202+0 \mathrm{i}$ & $-0.701-0 \mathrm{i}$ \\
\hline
\end{tabular}




\section{Time Domain Simulation Analysis}

In this section, the comparison among NSGA-II and NSSCA is presented based on the overshoot obtained from the step response of the inductor current (d-q), output voltage (dq), real power and reactive power for both DG-1 and DG-2 as shown in Figure 4. Considering the d-axis component of the inductor current of DG-1 as shown in Figure 4(a), the percentage of overshoot in the case of NSSCA is much less compared to NSGA-II. When, the d-axis component of the inductor current is considered for DG-2, NSGA-II results in $25 \%$ overshoot compared to zero overshoot in the case of

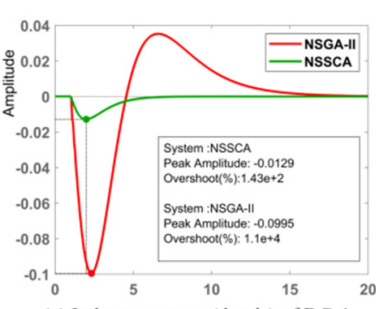

(a) Inductor current (d-axis) of DG-1

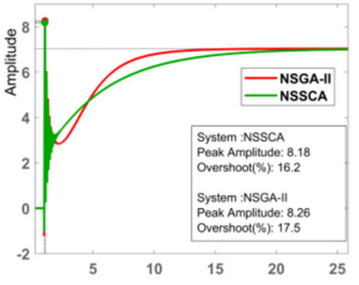

(e) Output voltage (d-axis) of DG-1

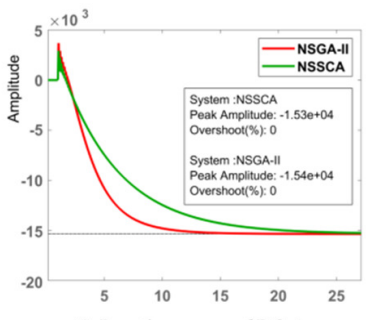

(i) Reactive power of DG-1

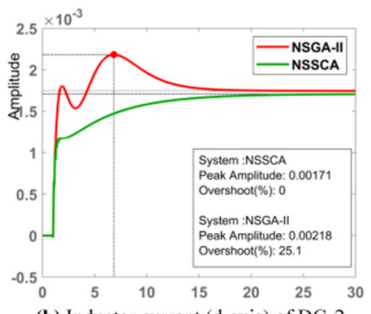

(b) Inductor current (d-axis) of DG-2

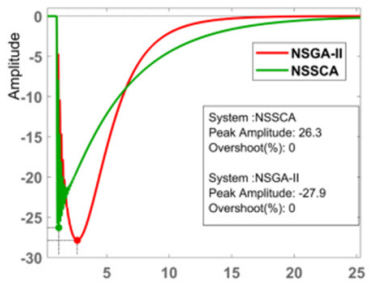

(f) Output voltage (d-axis) of DG-2

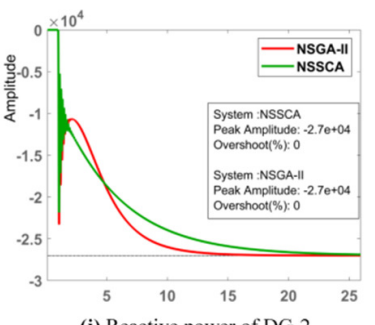

NSSCA. For both DG-1 and DG-2, when the step response of the q-axis component of the inductor current is considered, no overshoot is caused by NSGA-II and NSSCA. Considering the step response of the output voltages (d-q) of both DG-1 and DG-2 as shown in Figure 4(e)-(h), it can be observed that NSSCA causes less overshoot than NSGA-II with the only exception in case of the q-axis component of the output voltage of DG-2. For both DGs, the step response of the real and reactive power indicate that both the algorithms cause zero overshoot as depicted in Figure 4(i)-(1). In the light of the above discussion, it can be concluded that NSSCA provides better step response compared to NSGA-II.

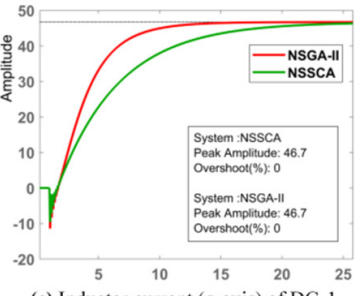

(c) Inductor current (q-axis) of DG-1

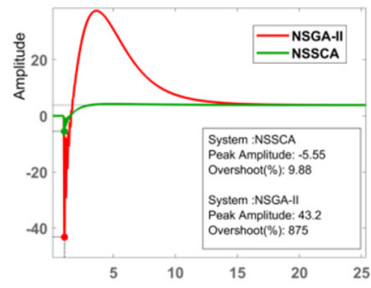

(g) Output voltage (q-axis) of DG-1

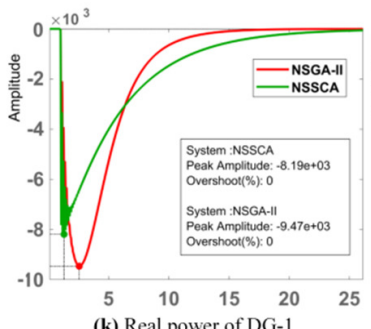

(k) Real power of DG-1

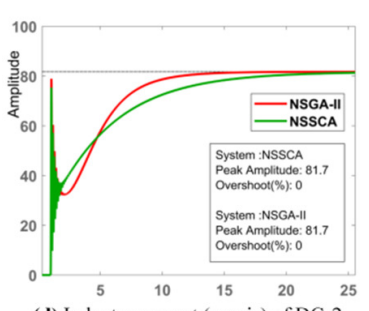

(d) Inductor current (q-axis) of DG-2

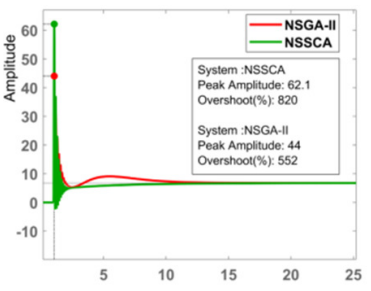

(h) Output voltage (q-axis) of DG-2

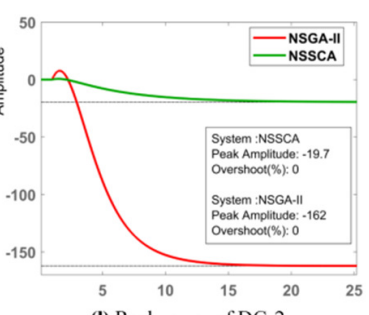

Fig. 4. Step response

\section{Statistical Tests}

In order to justify the uniqueness of each algorithm, independent samples' t-test was performed to compare the equality of means using SPSS [15] statistical analysis software. The t-test was performed with respect to the total number of iterations required to complete the optimization process, the total execution time of the optimization process, and the total summation of the real part of the eigenvalues. While running the t-test, SPSS software generated the results of the F-test which indicate whether the data samples from the two grouping variables (i.e. NSGA-II and NSSCA in this case) possess equal variances or not. For the F-test, the null hypothesis assumes that the data samples from the two groups have equal variances and the alternative hypothesis assumes that the data samples have non-equal variances. The null hypothesis can only be rejected when the significant factor ( $p$-value) of the F-test is less than 0.05 . From the results summarized in Table II, it can

be observed that the $p$-value of the F-test is greater than 0.05 only in case of the total summation of the real part of the eigenvalues. Thus, the data sets of NSGA-II and NSSCA possess non-equal variances in terms of total number of iterations and total execution time. The corresponding t-test results are also summarized in Table II. In the case of the t-test, the null hypothesis assumes that the means of two data sets are equal and as the alternative hypothesis it is assumed that the means of two data sets are not equal. If the significant factor ( $p$-value) of the t-test is less than 0.05 , then the null hypothesis can be rejected. From Table II it can be observed that there is a significant difference between the two algorithms with respect to the total number of iterations and the execution time as for both cases the p-value of the t-test is less than 0.05 , whereas, with respect to the total summation of eigenvalues the null hypothesis cannot be rejected as the p-value of the t-test is greater than 0.05 . However, from the data presented in Table 
III, the total summation of the eigenvalues in the case of NSSCA is slightly higher compared to NSGA-II which indicates that NSSCA ensures slightly better stability to the system. From the above analysis, it can be concluded that each algorithm possesses unique characteristics. Considering the mean values of the total number of iterations, total execution time, and total summation of eigenvalues of both algorithms, NSSCA was found to exhibit significantly better performance.

TABLE II. COMPARISON BETWEEN NSGA-II AND NSSCA BASED ON THE F-TEST AND T-TEST RESULTS

\begin{tabular}{|c|c|c|c|c|c|c|}
\hline \multirow{2}{*}{ Parameters } & \multicolumn{2}{|c|}{ F-test } & \multicolumn{3}{c|}{ t-test for equality of means } \\
\cline { 2 - 7 } & F & Sig. & $\begin{array}{c}\text { Mean } \\
\text { diff. }\end{array}$ & $\mathbf{t}$ & df & $\begin{array}{c}\text { Sig. } \\
\text { (2-tailed) }\end{array}$ \\
$\begin{array}{c}\text { Total } \\
\text { iterations }\end{array}$ & 60.035 & 0.000 & 10.133 & 6.177 & 29.273 & 0.000 \\
\hline $\begin{array}{c}\text { Total } \\
\text { summation of } \\
\text { eigenvalues } \\
\text { (real) }\end{array}$ & 2.477 & 0.121 & 30941 & 1.356 & 58 & 0.180 \\
\hline $\begin{array}{c}\text { Execution time } \\
\text { (n) }\end{array}$ & 38.545 & 0.000 & 3.043 & 2.155 & 33.202 & 0.039 \\
\hline
\end{tabular}

TABLE III. GROUP STATISTICAL DATA OF NSGA-II AND NSSCA

\begin{tabular}{|c|c|c|c|c|}
\hline \multirow{2}{*}{ Parameters } & Algorithm & Mean & $\begin{array}{c}\text { Standard } \\
\text { deviation }\end{array}$ & $\begin{array}{c}\text { Standard } \\
\text { error mean }\end{array}$ \\
\hline $\begin{array}{c}\text { Total number of } \\
\text { iterations }\end{array}$ & NSGA-II & 12 & 8.965 & 1.637 \\
\cline { 2 - 5 } & NSSCA & 2 & 0.615 & 0.112 \\
\hline $\begin{array}{c}\text { Total summation of } \\
\text { eigenvalues (real) }\end{array}$ & NSGA-II & -12709275 & 68444 & 12496 \\
\cline { 2 - 5 } & NSSCA & -12740216 & 104560 & 19090 \\
\hline \multirow{2}{*}{ Execution time } & NSGA-II & 10.660 & 7.468 & 1.363 \\
\cline { 2 - 5 } & NSSCA & 7.617 & 2.015 & 0.368 \\
\hline
\end{tabular}

\section{CONCLUSION}

In this study, the non-dominated sorting technique was merged with the Sine Cosine Algorithm (SCA) in order to develop a multi-objective optimization algorithm named NSSCA. This algorithm was applied to optimize the controller gains of a two bus microgrid model. The microgrid model was developed considering static load in one of the buses and an induction motor as dynamic load in the other bus. The performance of NSSCA in optimizing the controller gains was compared with NSGA-II by applying NSGA-II for the same two-bus system. From the comparative study in terms of eigenvalue analysis, time domain analysis, and statistical tests it was observed that NSSCA performs better in stabilizing the system by optimizing controller gains. The computations done by NSSCA were significantly faster compared to NSGA-II in terms of both required number of iterations and execution time. Thus, NSSCA can be considered as a prospective algorithm in optimizing the controller gains of a microgrid model.

\section{REFERENCES}

[1] A. M. Howlader et al., "A minimal order observer based frequency control strategy for an integrated wind-battery-diesel power system," Energy, vol. 46, no. 1, pp. 168-178, Oct. 2012, doi: 10.1016/j.energy.2012.08.039.

[2] N. Pogaku, M. Prodanovic, and T. C. Green, "Modeling, Analysis and Testing of Autonomous Operation of an Inverter-Based Microgrid," IEEE Transactions on Power Electronics, vol. 22, no. 2, pp. 613-625, Mar. 2007, doi: 10.1109/TPEL.2006.890003.
[3] L. S. Coelho and V. C. Mariani, "Combining of chaotic differential evolution and quadratic programming for economic dispatch optimization with valve-point effect," IEEE Transactions on Power Systems, vol. 21, no. 2, pp. 989-996, May 2006, doi: 10.1109/TPWRS.2006.873410.

[4] R. Eslami, S. H. H. Sadeghi, and H. A. Abyaneh, "A Probabilistic Approach for the Evaluation of Fault Detection Schemes in Microgrids," Engineering, Technology \& Applied Science Research, vol. 7, no. 5, pp. 1967-1973, Oct. 2017.

[5] S. Sinha and S. S. Chandel, "Review of recent trends in optimization techniques for solar photovoltaic-wind based hybrid energy systems," Renewable and Sustainable Energy Reviews, vol. 50, pp. 755-769, Oct. 2015, doi: 10.1016/j.rser.2015.05.040.

[6] E. E. Miandoab and F. S. Gharehchopogh, "A Novel Hybrid Algorithm for Software Cost Estimation Based on Cuckoo Optimization and KNearest Neighbors Algorithms," Engineering, Technology \& Applied Science Research, vol. 6, no. 3, pp. 1018-1022, Jun. 2016.

[7] K. Prabakar, F. Li, and B. Xiao, "Controller hardware-in-loop testbed setup for multi-objective optimization based tuning of inverter controller parameters in a microgrid setting," in 2016 Clemson University Power Systems Conference (PSC), Clemson, SC, USA, Mar. 2016, doi: 10.1109/PSC.2016.7462824.

[8] M. Fadaee and M. A. M. Radzi, "Multi-objective optimization of a stand-alone hybrid renewable energy system by using evolutionary algorithms: A review," Renewable and Sustainable Energy Reviews, vol. 16, no. 5, pp. 3364-3369, Jun. 2012, doi: 10.1016/j.rser.2012.02.071.

[9] A. Ibrahim, Y. Jibril, and Y. S. Haruna, "Determination of Optimal Droop Controller Parameters for an Islanded Microgrid System Using Artificial Fish Swarm Algorithm (AFSA)," International Journal of Scientific \& Engineering Research, vol. 8, no. 3, pp. 959-965, Mar. 2017.

[10] R. Wang et al., "Optimized Operation and Control of Microgrid based on Multi-objective Genetic Algorithm," in 2018 International Conference on Power System Technology (POWERCON), Guangzhou, China, Nov. 2018, pp. 1539-1544, doi: 10.1109/POWERCON.2018.8601845.

[11] S. Mirjalili, "SCA: A Sine Cosine Algorithm for solving optimization problems," Knowledge-Based Systems, vol. 96, pp. 120-133, Mar. 2016, doi: 10.1016/j.knosys.2015.12.022.

[12] K. Deb, A. Pratap, S. Agarwal, and T. Meyarivan, "A fast and elitist multiobjective genetic algorithm: NSGA-II," IEEE Transactions on Evolutionary Computation, vol. 6, no. 2, pp. 182-197, Apr. 2002, doi: 10.1109/4235.996017.

[13] A. Kahrobaeian and Y. A.-R. I. Mohamed, "Analysis and Mitigation of Low-Frequency Instabilities in Autonomous Medium-Voltage Converter-Based Microgrids With Dynamic Loads," IEEE Transactions on Industrial Electronics, vol. 61, no. 4, pp. 1643-1658, Apr. 2014, doi: 10.1109/TIE.2013.2264790.

[14] S. R. Mudaliyar and S. S. Sahoo, "Comparison of different eigenvalue based multi-objective functions for robust design of power system stabilizers," International Journal of Electrical and Electronic Engineering \& Telecommunications, vol. 1, no. 2, 2015.

[15] SPSS Inc. Released 2007. SPSS for Windows, Version 16.0. Chicago, SPSS Inc. 